\title{
Metabolomics reveals stage-specific metabolic pathways of microbial communities in two-stage anaerobic fermentation of corn-stalk
}

\author{
Dawei Yang $\cdot$ Xiaolei Fan $\cdot$ Xiaoshuang Shi $\cdot$ \\ Shujuan Lian · Jiangtao Qiao · Rongbo Guo
}

Received: 12 December 2013/Accepted: 3 March 2014/Published online: 22 March 2014

(C) Springer Science+Business Media Dordrecht 2014

\begin{abstract}
Analysis of intracellular metabolites is essential to delineate metabolic pathways of microbial communities for evaluation and optimization of anaerobic fermentation processes. The metabolomics are reported for a microbial community during two stages of anaerobic fermentation of corn stalk in a biogas digester using GC-MS. Acetonitrile/methanol/ water (2:2:1, by vol) was the best extraction solvent for microbial community analysis because it yielded the largest number of peaks ( $>200)$, the highest mean summed value of identified metabolites (23) and the best reproducibility with a coefficient of variation of $30 \%$ among four different extraction methods. Inter-
\end{abstract}

Electronic supplementary material The online version of this article (doi:10.1007/s10529-014-1508-3) contains supplementary material, which is available to authorized users.

D. Yang $\cdot$ X. Fan $(\bowtie) \cdot$ X. Shi $\cdot$ S. Lian .

J. Qiao · R. Guo ( $₫)$

Key Laboratory of Biofuels, Shandong Industrial Engineering Laboratory of Biogas Production \& Utilization, Qingdao Institute of Bioenergy and Bioprocess Technology, Chinese Academy of Sciences, No. 189 Songling Road, Qingdao 266101, Shandong, China

e-mail: fanxl@qibebt.ac.cn

R. Guo

e-mail: guorb@qibebt.ac.cn

D. Yang

Central Laboratory, Liaocheng People's Hospital,

Liaocheng 252000, Shandong, China stage comparison of metabolite profiles showed increased levels of sugars and sugar alcohols during methanogenesis and fatty acids during acidogenesis. Identification of stage-specific metabolic pathways using metabolomics can therefore assist in monitoring and optimization of the microbial community for increased biogas production during anaerobic fermentation.

Keywords Anaerobic fermentation · Corn stalk $\cdot$ Metabolomics $\cdot$ Microbial communities

\section{Introduction}

Cellulosic biomass is used to produce biofuels by microbial fermentation (Levin et al. 2007; Gunaseelan 1997). In China about 700 million tons of cellulosic biomass are produced each year, of which around 100 million tons is corn stalk (Fan et al. 2008; Wang et al. 2010). Development of an advanced anaerobic fermentation process of corn stalk is under active study to improve its efficient utilization for methane production.

Metagenomic analysis of microbial communities have suggested that a range of metabolic genes are active in biogas digester processes (Handelsman 2004; Riesenfeld et al. 2004; Tringe et al. 2005). Although the presence of genes in microbial communities suggests possible metabolic trajectories, quantitative measurements of metabolites and proteins are required 
to reveal the metabolic pathways activated in different fermentation stages.

Metabolomics is the comprehensive, non-biased analysis of all the metabolites that contribute to the molecular interactions and metabolic pathways in biological systems (Bino et al. 2004; Fiehn 2002). Microbial communities have strong relationships between members of the community and their function rather than the environment during anaerobic digestion (Werner et al. 2011). Therefore, it is possible to explore the relationship between metabolism of a microbial community and its overall activity (e.g. methanogenic activity) using a metabolomics approach. To date, no metabolomics studies have been reported on the microbial community using corn stalks in an anaerobic fermentation.

The present study assessed four different extraction solvents that are the most widely used in microbial metabolomics studies. The optimized metabolomics method was then used to investigate two-stage corn stalk fermentation, a methane production method that separates the hydrolysis/acidogenesis stage from the methanogenesis stage, resulting in improved biogas yield compared with single-stage fermentation approach (Blonskaja et al. 2003; Liu et al. 2006; Lu et al. 2009). This approach should therefore reveal relationships between variations in metabolism and the different fermentation process improving our understanding of microbial community regulation and function.

\section{Materials and methods}

Parameters of the continuous stirred tank

A continuous stirred tank (CSTR) reactor with a working volume of 41 was operated at $35 \pm 1{ }^{\circ} \mathrm{C}$. The reactor was stirred with a blender for $10 \mathrm{~min}$ every $3 \mathrm{~h}$. At start-up, the reactor was filled with $35 \mathrm{~g}$ total solid (TS) $1^{-1}$ cattle manure as the inoculum. The

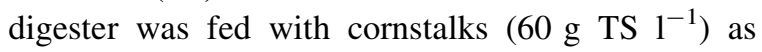
substrate. The reactor performance (biogas-methaneproduction and $\mathrm{pH}$ ) was monitored daily. A steady state was assumed when the variation of the monitoring parameters over time was less than $10 \%$. The daily biogas production rate was 0.98 days $1^{-1}$ at a hydraulic retention time (HRT) of 20 days. Samples for optimization of metabolomics sample preparation were obtained from an outlet pipe attached to the CSTR reactor during steady state operation.

Two-stage fermentation process

The sun-cured cornstalk was milled to $5 \mathrm{~mm}$. The total solid (TS) and volatile solid (VS) contents of cornstalk were 94 and $85 \%$, respectively. The TS and VS of manure were 17 and $13 \%$, respectively. Prior to feeding to the reactors, both substrates were heated at $100{ }^{\circ} \mathrm{C}$ for $15 \mathrm{~min}$ to inhibit the activity of methanogenic and other pathogenic microbes and to enrich the $\mathrm{H}_{2}$-producing bacteria.

The two-stage process consisted of two reactors, an acidogenic reactor and a methanogenic reactor, with working volume of 1 and 21 , respectively. Both reactors were maintained at $37 \pm 1{ }^{\circ} \mathrm{C}$. The acidogenic reactor was operated for 6 days in a batch mode. The $\mathrm{pH}$ and COD of the acidogenic liquid were 5.1 and $8.7 \mathrm{~g} \mathrm{l}^{-1}$, respectively. The acidogenic liquid was fed into the methanogenic reactor by a peristaltic pump. The $\mathrm{pH}$ was $7.2 \pm 0.2$ and the methane content was $60 \pm 5 \%$ with a HRT of 10 days in the methanogenic reactor. Six replicate samples were collected during steady state operation of the acidogenic and methanogenic fermentation stages for metabolomics analysis.

\section{Quenching}

Ten $\mathrm{ml}$ of fermentation broth was sprayed into $10 \mathrm{ml}$ quenching solution (methanol/water, 3:2 v/v) at $-50{ }^{\circ} \mathrm{C}$ (Winder et al. 2008). The solution was rapidly filtered through a membrane $(30-50 \mu \mathrm{m})$ to remove corn stalk debris. The samples were centrifuged at $10,000 \times g$ for $5 \mathrm{~min}$ at $4{ }^{\circ} \mathrm{C}$. Cell pellets were snapfrozen in liquid $\mathrm{N}_{2}$ and stored at $-80{ }^{\circ} \mathrm{C}$ for further extraction.

\section{Extraction of intracellular metabolites}

After quenching, the cell pellets were subjected to different metabolite extraction methods. Four different protocols for extraction of intracellular metabolites were tested using six technical replicate samples.

Cold methanol/water

We modified a procedure described Duportet et al. (2012). Briefly, the cell pellets were re-suspended in 
$2.5 \mathrm{ml}$ methanol/water $(1: 1, \mathrm{v} / \mathrm{v})$ at $-20{ }^{\circ} \mathrm{C}$. Each sample was vortexed vigorously for $30 \mathrm{~s}$. The mixed samples were then frozen at $-80{ }^{\circ} \mathrm{C}$ and subjected to three freeze-thaw cycles of 2 min for each cycle after which the samples were centrifuged at $10,000 \times g$ at $4{ }^{\circ} \mathrm{C}$.

$100 \%$ methanol coupled to sonication

The cell pellets were re-suspended in $2.5 \mathrm{ml}$ methanol at $-20{ }^{\circ} \mathrm{C}$ and vortexed for $30 \mathrm{~s}$. The re-suspended samples were sonicated for $1 \mathrm{~min}$ in an ice bath with a sonicator operating at $20 \mathrm{kHz}$. The samples were then centrifuged at $10,000 \times g$ at $4{ }^{\circ} \mathrm{C}$.

Acidic solvent mixtures

The cell pellets were extracted with $2.5 \mathrm{ml}$ acetonitrile/methanol/water (2:2:1, by vol) or water/2-propanol/methanol $\left(2: 2: 5\right.$, by vol) at $-20{ }^{\circ} \mathrm{C}$ according to Kim et al. (2013) and Shin et al. (2010). After centrifugation, all the extraction solution and the supernatant were concentrated to dryness in a vacuum concentrator. The residue was re-suspended in $500 \mu \mathrm{l}$ acetonitrile/water $(1: 1, \mathrm{v} / \mathrm{v})$ to remove complex lipids and waxes. After centrifugation at $16,000 \times g$ for $5 \mathrm{~min}$, the supernatant was concentrated to dryness again and kept at $-80{ }^{\circ} \mathrm{C}$ prior to derivatization.

\section{Sample derivatization}

The residue was dissolved in $20 \mu 198 \%$ methoxyamine hydrochloride $\left(40 \mathrm{mg} \mathrm{ml}^{-1}\right)$ in pyridine and shaken at $30{ }^{\circ} \mathrm{C}$ for $90 \mathrm{~min}$. Then, $90 \mu \mathrm{l}$-methyl- $N$ (trimethylsilyl) trifluoroacetamide (MSTFA) was added for trimethylsilylation of acidic protons and shaken at $37{ }^{\circ} \mathrm{C}$ for $30 \mathrm{~min}$.

\section{GC-MS analysis}

GC-MS analysis was carried out using a $30 \mathrm{~m} \times$ $0.25 \mathrm{~mm} \times 0.25 \mu \mathrm{m}$ DB-5 MS column (J\&W Scientific, CA, USA). Helium was used as carrier gas at $1 \mathrm{ml} / \mathrm{min}$. Derivatized samples $(1 \mu \mathrm{l})$ were injected at splitless mode. The oven was held at $100{ }^{\circ} \mathrm{C}$ for $3 \mathrm{~min}$, then ramped by $5^{\circ} \mathrm{C} \min ^{-1}$ to $320{ }^{\circ} \mathrm{C}$, and held for $10 \mathrm{~min}$. The ionization was processed in EI positive mode. Ion source and quadrupole temperature were set to 230 and $150{ }^{\circ} \mathrm{C}$, respectively. Mass spectra were acquired in a scan range of $50-500 \mathrm{~m} / \mathrm{z}$. All samples were prepared and analyzed in random order.

\section{Data processing}

GC-MS data were exported from Agilent MSD ChemStation into netCDF format. The Automated Mass Spectral Deconvolution and Identification System (AMDIS) was used to deconvolute the netCDF files in batch mode. AMDIS deconvolution settings were as follows: resolution was medium, sensitivity was low, shape requirement was medium and component width was set at 10 . The *.ELU files generated from AMDIS were uploaded to the SpectConnect online service (http://spectconnect.mit.edu/) to find the complement of peaks that are consistently detected in multiple chromatograms of one of the study design classes (e.g. per extraction method) (Barupal et al. 2010; Styczynski et al. 2007). After data was pre-processed by SpectConnect, four matrices, including RA, RT, IS and AM (for relative amount, retention time, integrated peak and base peak) can be acquired for further multivariate statistics. Peak identification was achieved in AMDIS to search the mass spectra against the NIST mass spectral library (consisting of NIST 08, Wiley, Replib and Fiehn Library) (Kind et al. 2009).

Multivariate statistical analysis was performed with a web-based analytical pipeline Metaboanalyst (Xia et al. 2012). One-way analysis of variance (ANOVA) was achieved by Statistica (Statsoft Tulsa). Metabolic networks were performed by MetaMapp (Barupal et al. 2012), which uses KEGG reaction pairs to build a metabolic network and then adds non-mapped compounds via Tanimoto similarity.

\section{Results and discussion}

Different metabolic profiles from different extraction methods

We observed different metabolite profiles were generated from the four extraction methods as confirmed by PCA analysis of the data matrix (see Fig. 1a). The differences in the metabolite profiles explained by the first principal component, $46.8 \%$. Samples from the same extraction methods were clustered together. PCA analysis showed that extraction with acetonitrile/ 
$\mathbf{a}$

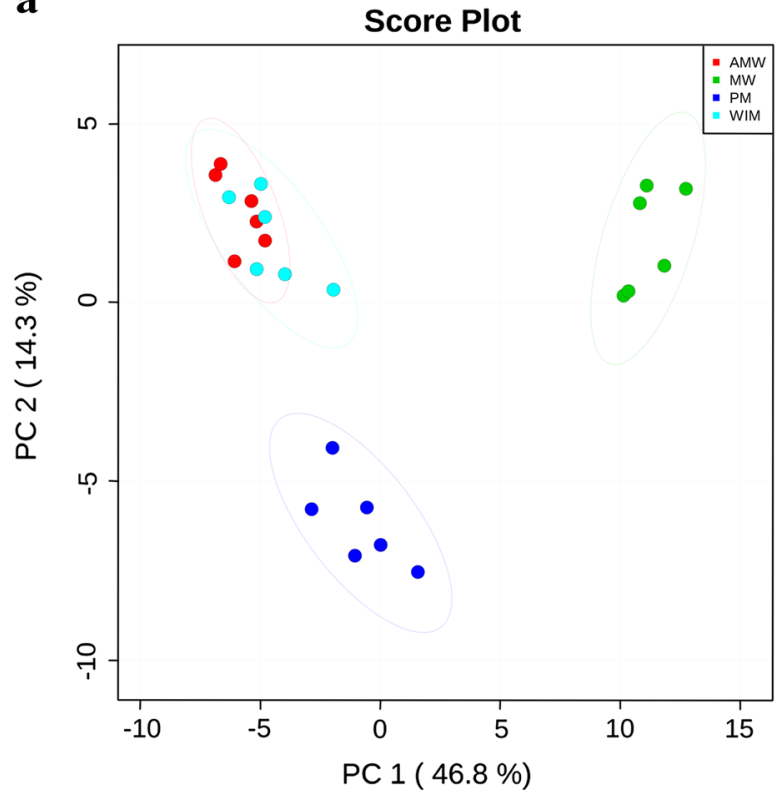

Fig. 1 Score plot shows the response of intracellular metabolites to four extraction methods using PCA a and the clustered heatmap of intracellular metabolites of four extraction methods (b). Green represents compounds detected at low concentration.

methanol/water mixture (AMW) and water/2-propanol/methanol (WIM) presented similar profiles but they were different from the profiles generated using methanol/water or $100 \%$ methanol.

\section{Optimization of extraction method}

Extraction with AMW generated the highest numbers of peaks among the extraction methods tested (see Table 1). WIM generated the second highest number of peaks, followed by $100 \%$ methanol while methanol/water gave the smallest number of peaks. The number of peaks detected was counted in the deconvolution data after processing by SpectConnect. Extracting the largest number and diversity of metabolites is the most important criterion for the extraction solvent, so AMW and WIM were the best extraction solvents because of the larger pool of metabolites detected compared with the other two solvents. Supplementary Fig. 1 shows a box plot of some representative metabolites based on ANOVA analysis showing specific trends in metabolite differences obtained with different extraction methods. A diverse range of metabolites, including xylitol, succinic acid, stearic acid, proline, acetic acid, inositol, glycerol

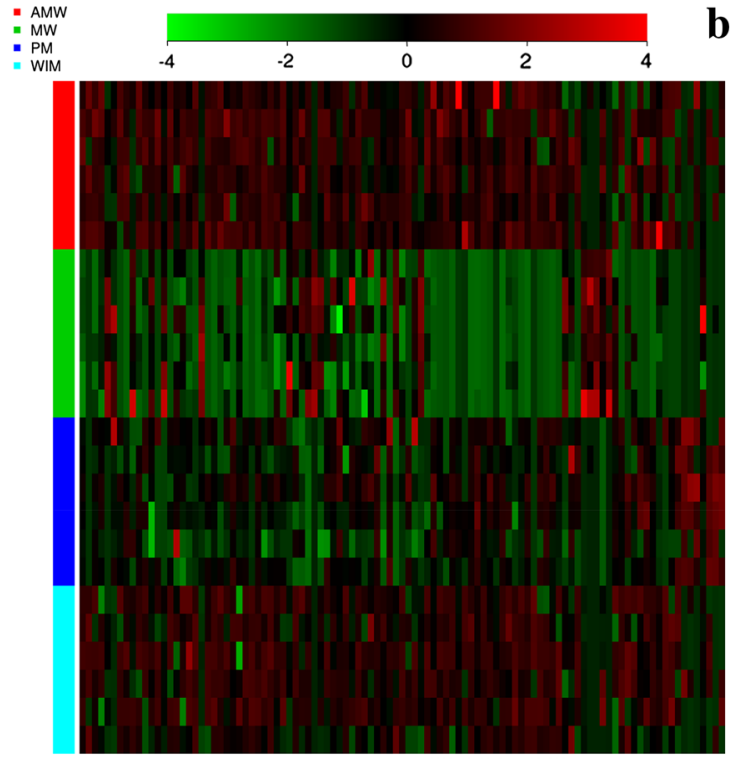

Red represents compounds detected at high concentrations. MW, methanol/water (1:1 v/v); PM, $100 \%$ methanol; AMW, acetonitrile/methanol/water $(2: 2: 1$, by vol); WIM, water/2propanol/methanol $(2: 2: 5$, by vol)

Table 1 Comparison of the number of peaks, mean summed value of identified metabolites and coefficient of variation when different extraction solvents were used

\begin{tabular}{lcll}
\hline $\begin{array}{l}\text { Extraction } \\
\text { method }\end{array}$ & $\begin{array}{l}\text { Number of } \\
\text { peaks }^{\mathrm{a}}\end{array}$ & $\begin{array}{l}\text { Mean summed } \\
\text { value }\end{array}$ & $\begin{array}{l}\mathrm{CV}^{\mathrm{b}} \\
(\%)\end{array}$ \\
\hline $\mathrm{MW}^{\mathrm{c}}$ & $163.5 \pm 13.9$ & -21.1 & 46.4 \\
$\mathrm{M}^{\mathrm{d}}$ & $178 \pm 12.8$ & -14.3 & 43.3 \\
$\mathrm{AMW}^{\mathrm{e}}$ & $212.3 \pm 9.7$ & 23 & 30 \\
$\mathrm{WIM}^{\mathrm{f}}$ & $206.8 \pm 8.8$ & 12.3 & 35.5 \\
\hline
\end{tabular}

${ }^{a}$ Six replicates were analyzed for each extraction method and expressed as the mean \pm standard deviation

b Coefficient of variation

c Methanol/water (1:1, v/v)

d Methanol (100\%)

e Acetonitrile/methanol/water (2:2:1, by vol)

${ }^{f}$ Water/2-propanol/methanol (2:2:5, by vol)

3-phosphate, mannose, and mannitol were efficiently extracted with AMW and WIM.

The second criterion for the selection of the optimal extraction method is the peak intensities of each metabolite. A heat-map of the intracellular metabolites obtained from each of four extraction methods is shown in Fig. 1b. WIM and AMW methods generated more and higher peaks of high concentration than the 
other two methods. Peak intensity of each metabolite, normalized by autoscaling method (Shin et al. 2010; van den Berg et al. 2006), subtracted by its mean and then divided by its standard deviation, was used to evaluate the four extraction methods as the second criterion. The mean summed value of identified metabolites for methanol/water, methanol only, AMW and WIM were $-21.1,-14.3,23$ and 12.3 (see Table 1), respectively. The reproducibility of each extraction method was assessed on the coefficient of variation $(\mathrm{CV})$ of all metabolites. AMW showed the highest reproducibility for the total metabolites with a $\%$ CV of 30 (see Table 1), followed by WIM. Methanol/water had the highest median CV, indicating the lowest reproducibility with respect to the extraction yield of metabolites. Overall, the best performance, based on three criteria, was achieved with AMW, which yielded the largest number of peaks, the highest peak intensities of identified metabolites and the best reproducibility among four different extraction methods.
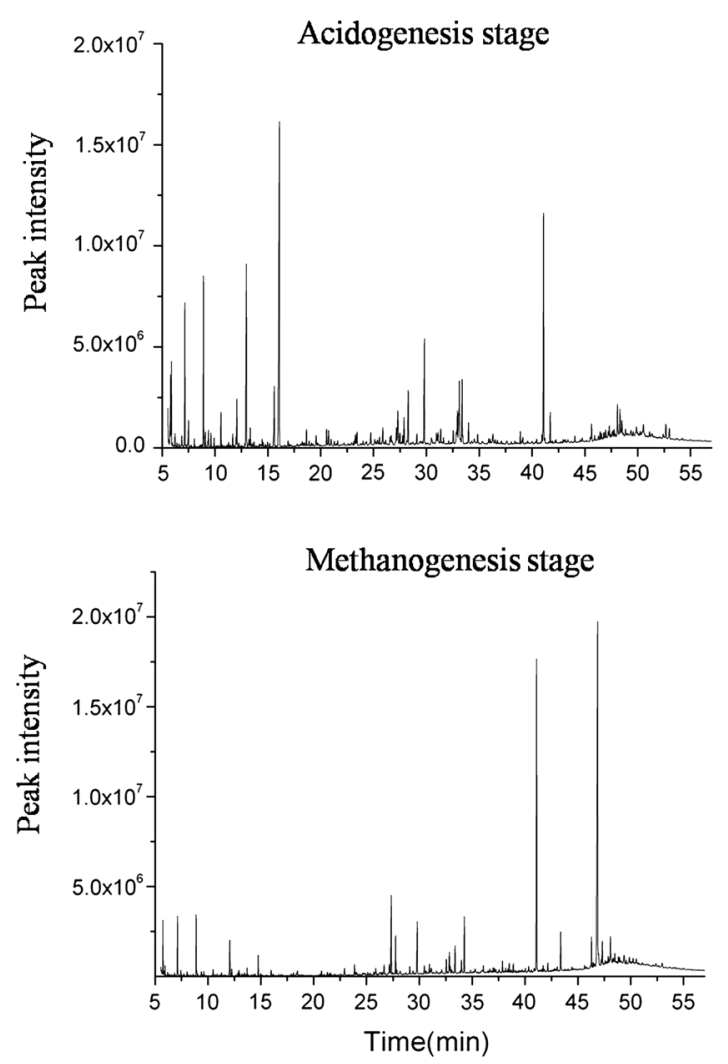

In previous microbial metabolomics studies, cold methanol was selected as the best extraction solvents for Escherichia coli (Winder et al. 2008), yeast (Villas-Bôas et al. 2005) and Lactobacillus plantarum (Faijes et al. 2007). Acidic acetonitrile/methanol/water mixture was also recommended for extraction of the E. coli metabolome (Rabinowitz and Kimball 2007). Acetonitrile/ water $(1: 1, \mathrm{v} / \mathrm{v})$ or boiling ethanol $(75 \%, \mathrm{v} / \mathrm{v})$ was the most effective extraction solvent for Saccharomyces cerevisiae (Kim et al. 2013). In contrast, acidic acetonitrile/methanol, compared with boiling ethanol and chloroform/methanol, was not suitable for extracting the metabolome of yeast (Canelas et al. 2009). Therefore, the extraction method needs to be optimized for each microorganism and especially for microbial communities that contain different bacteria and archaea.

\section{Metabolic shift in two-stage fermentation}

We applied the optimized metabolomics method for the analysis of metabolic alterations in the two-stage

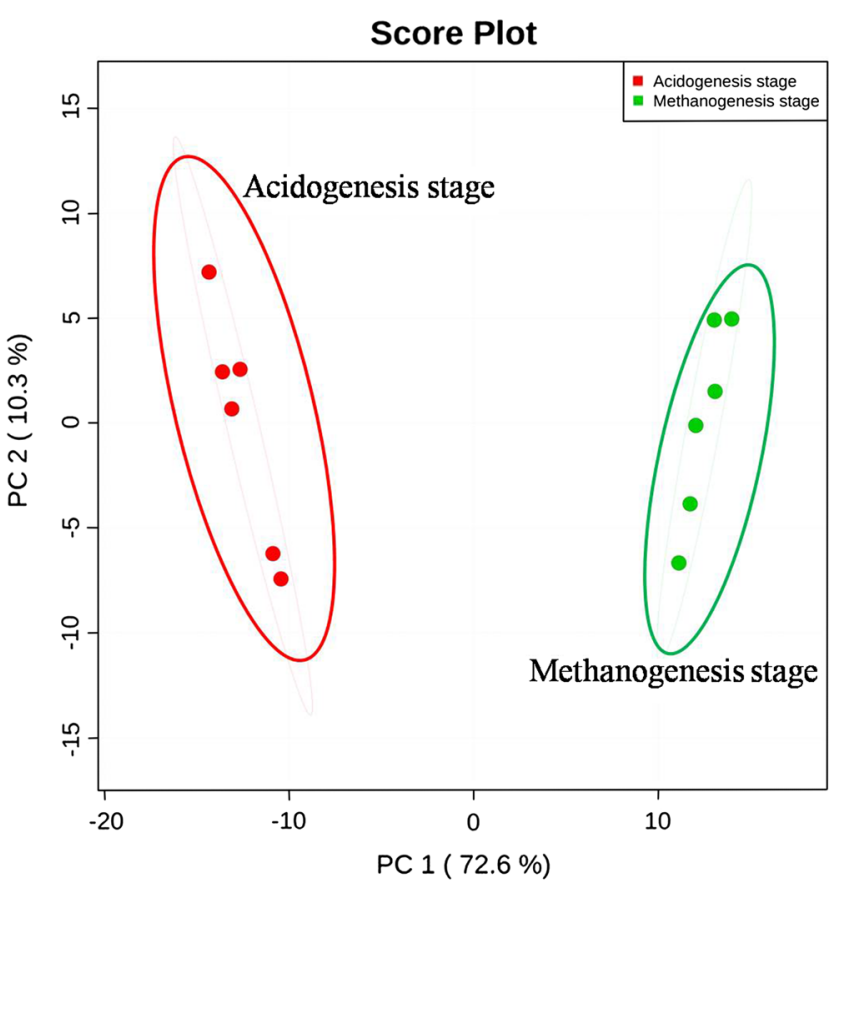

Fig. 2 Typical chromatograms of intracellular metabolites profile during acidogenesis and methanogenesis, and PCA analysis of metabolic response during the two-stage fermentation process 
fermentation of corn stalk. A total of 238 peaks (86 knowns and 152 unknowns) were detected by GC-MS (Supplementary Table 1). Typical chromatograms of different fermentation stages showed different metabolite profiles (Fig. 2). The acidogenesis stage contained more peaks than the methanogenesis stage during the first $30 \mathrm{~min}$, which meant that more small molecular weight metabolites were detected in the acidogenesis stage. GC-MS data were subjected to Metaboanalyst to perform PCA analysis by normalizing metabolite concentrations to their DNA content (see Supplementary Methods: $2 \pm 0.75 \mu \mathrm{g} \mathrm{ml}^{-1}$ for the acidogenesis stage and $6.86 \pm 1.45 \mu \mathrm{g} \mathrm{ml}^{-1}$ for the methanogenesis stage). The PCA score plot readily distinguished between acidogenesis and methanogenesis based on the metabolite profiles (Fig. 2).

Mapping metabolomics data is highlighted in Fig. 3 for the two-stage fermentation process, displaying significantly altered metabolites while not labeling compounds that were unchanged. Fatty acids and fatty acid esters were detected at higher concentration during acidogenesis stage, whereas sugars and sugar alcohols were more abundant during methanogenesis than acidogenesis (Fig. 3). Surprisingly, most of the sugars and sugar alcohols were not detected during acidogenesis stage. Most amino acids and amines, except leucine and isoleucine, were detected at higher concentrations during acidogenesis but not methanogenesis.

In the acidogenesis stage, methanogenic archaea were inactivated by the heating procedure, therefore, the main microorganisms functioning during acidogenesis were hydrolytic and fermentative microorganisms digesting cellulose, protein and fat into sugar, amino acids, fatty acids and other small metabolites. Concentrations of fatty acids and amino acids increased during acidogenesis, validating the use of the metabolomics method of this study. The absence of sugars and sugar alcohols during acidogenesis suggested that microbial communities without archaea consumed sugars derived from cellulose. Because of the large amount of sugars and sugar alcohols in methanogenesis stage, we infer that the metabolic functions of the microbial community during methanogenesis

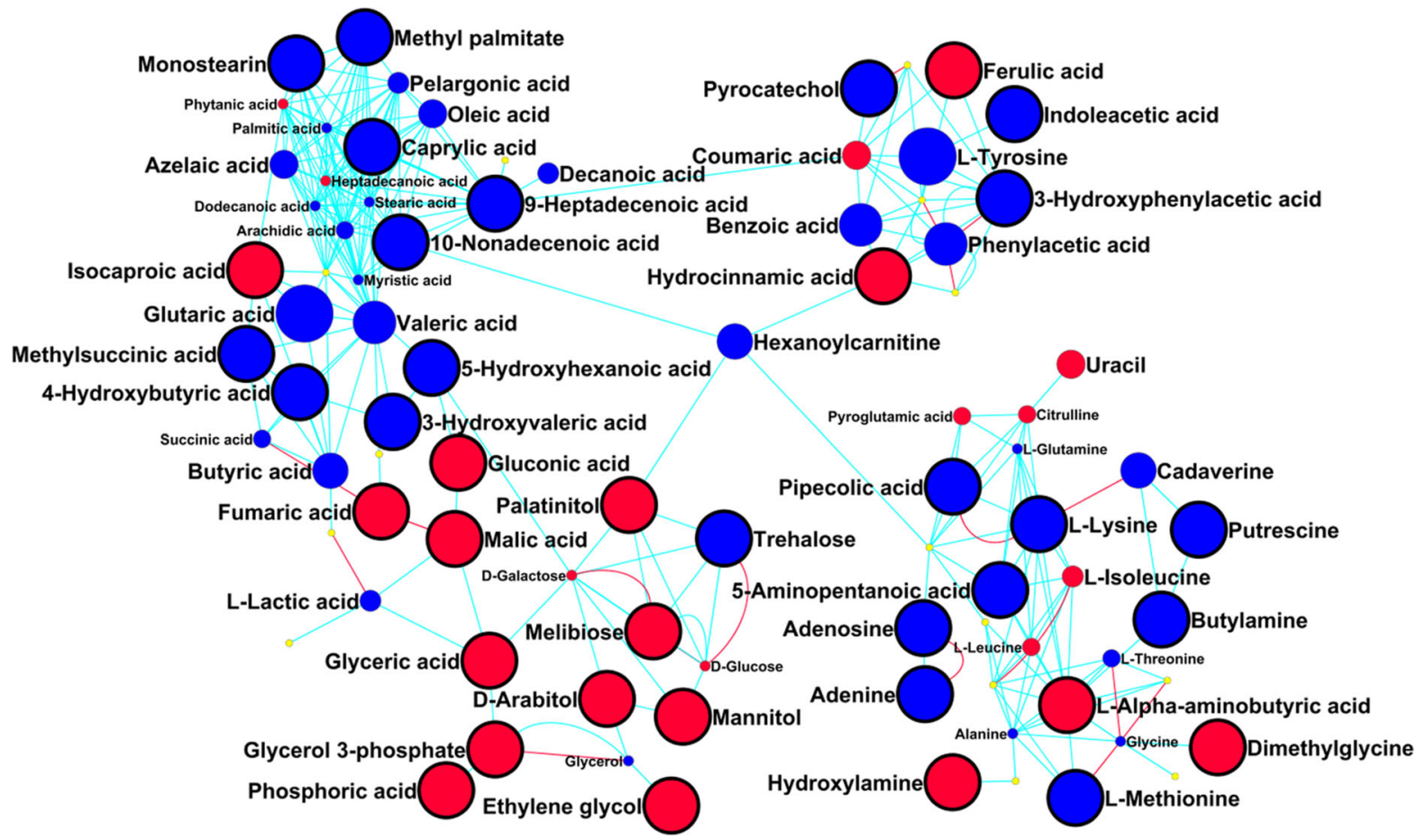

Fig. 3 MetaMapp visualization of metabolomics data in the two-stage fermentation process. Red edges denote KEGG reactant pair links; blue edges symbolize Tanimoto chemical similarity at $\mathrm{T}>700$; Blue nodes represent up-regulated metabolites during acidogenesis; Red nodes represent upregulated metabolites during methanogenesis; Nodes with borders represent metabolites detected only in one stage; Node size reflects fold change 
differ from those during acidogenesis. During methanogenesis a new microbial community was formed with both anaerobic bacteria and archaea. The limited number of small molecular weight metabolites during methanogenesis reflects the fact that those were utilized by methanogenic archaea for methane production, while not all of the sugars and sugar alcohols were used at that time. This explains why we observed both sugars and small metabolites during methane production in the methanogenesis stage. Overall, the metabolic changes reflect the shift in composition of the microbial community in the different fermentation stages. Metabolomics was used to establish the relationship between metabolites and biogas production of the biogas reactor. Therefore, the metabolomics approach is a promising method to monitor the status of biogas reactors by analyzing the intracellular metabolites from microbial communities.

\section{Conclusions}

This is the first study of the metabolome of the microbial community during corn stalk anaerobic digestion. The strategy presented in this study may shed light on development of combination of metabolomics and metagenomics in microbial community research providing important information for improving biogas yield.

Acknowledgments We thank Dinesh Kumar Barupal for helping the generation of metabolic network and the preparation of manuscript. This work were financial supported by the Natural Science Foundation of China (No. 41276143, 31101918), 863 program (No. 2012AA052103), National Science \& Technology Pillar Program (No. 2010BAC67B03), R\&D of biogas industry technology (No. Y113031103) and Taishan Scholar Program of Shandong.

\section{References}

Barupal D, Kind T, Kothari S, Lee D, Fiehn O (2010) Hydrocarbon phenotyping of algal species using pyrolysis-gas chromatography mass spectrometry. BMC Biotechnol $10: 40$

Barupal D, Haldiya P, Wohlgemuth G, Kind T, Kothari S, Pinkerton K, Fiehn O (2012) MetaMapp: mapping and visualizing metabolomic data by integrating information from biochemical pathways and chemical and mass spectral similarity. BMC Bioinform 13:99

Bino RJ, Hall RD, Fiehn O, Kopka J, Saito K, Draper J, Nikolau BJ, Mendes P, Roessner-Tunali U, Beale MH (2004)
Potential of metabolomics as a functional genomics tool. Trends Plant Sci 9:418-425

Blonskaja V, Menert A, Vilu R (2003) Use of two-stage anaerobic treatment for distillery waste. Adv Environ Res 7:671-678

Canelas AB, ten Pierick A, Ras C, Seifar RM, van Dam JC, van Gulik WM, Heijnen JJ (2009) Quantitative evaluation of intracellular metabolite extraction techniques for yeast metabolomics. Anal Chem 81:7379-7389

Duportet X, Aggio RBM, Carneiro S, Villas-Bôas SG (2012) The biological interpretation of metabolomic data can be misled by the extraction method used. Metabolomics $8: 410-421$

Faijes M, Mars A, Smid E (2007) Comparison of quenching and extraction methodologies for metabolome analysis of Lactobacillus plantarum. Microb Cell Fact 6:27

Fan YT, Xing Y, Ma HC, Pan CM, Hou HW (2008) Enhanced cellulose-hydrogen production from corn stalk by lesser panda manure. Int J Hydrogen Energy 33:6058-6065

Fiehn O (2002) Metabolomics: the link between genotypes and phenotypes. Plant Mol Biol 48:155-171

Gunaseelan NV (1997) Anaerobic digestion of biomass for methane production: a review. Biomass Bioenerg 13:83114

Handelsman J (2004) Metagenomics: application of genomics to uncultured microorganisms. Microbiol Mol Biol Rev 68:669-685

Kim S, Lee DY, Wohlgemuth G, Park HS, Fiehn O, Kim KH (2013) Evaluation and optimization of metabolome sample preparation methods for Saccharomyces cerevisiae. Anal Chem 85:2169-2176

Kind T, Wohlgemuth G, Lee DY, Lu Y, Palazoglu M, Shahbaz S, Fiehn O (2009) FiehnLib: mass spectral and retention index libraries for metabolomics based on quadrupole and time-of-flight gas chromatography/mass spectrometry. Anal Chem 81:10038-10048

Levin DB, Zhu H, Beland M, Cicek N, Holbein BE (2007) Potential for hydrogen and methane production from biomass residues in Canada. Bioresour Technol 98:654-660

Liu D, Liu D, Zeng RJ, Angelidaki I (2006) Hydrogen and methane production from household solid waste in the twostage fermentation process. Water Res 40:2230-2236

Lu Y, Lai Q, Zhang C, Zhao H, Ma K, Zhao X, Chen H, Liu D, Xing XH (2009) Characteristics of hydrogen and methane production from cornstalks by an augmented two-or threestage anaerobic fermentation process. Bioresour Technol 100:2889-2895

Rabinowitz JD, Kimball E (2007) Acidic acetonitrile for cellular metabolome extraction from Escherichia coli. Anal Chem 79:6167-6173

Riesenfeld CS, Schloss PD, Handelsman J (2004) Metagenomics: genomic analysis of microbial communities. Annu Rev Genet 38:525-552

Shin MH, Lee DY, Liu KH, Fiehn O, Kim KH (2010) Evaluation of sampling and extraction methodologies for the global metabolic profiling of Saccharophagus degradans. Anal Chem 82:6660-6666

Styczynski MP, Moxley JF, Tong LV, Walther JL, Jensen KL, Stephanopoulos GN (2007) Systematic identification of conserved metabolites in GC/MS data for metabolomics and biomarker discovery. Anal Chem 79:966-973 
Tringe SG, Von Mering C, Kobayashi A, Salamov AA, Chen K, Chang HW, Podar M, Short JM, Mathur EJ, Detter JC (2005) Comparative metagenomics of microbial communities. Science 308:554-557

van den Berg RA, Hoefsloot HC, Westerhuis JA, Smilde AK, van der Werf MJ (2006) Centering, scaling, and transformations: improving the biological information content of metabolomics data. BMC Genomics 7:142

Villas-Bôas SG, Højer-Pedersen J, Åkesson M, Smedsgaard J, Nielsen J (2005) Global metabolite analysis of yeast: evaluation of sample preparation methods. Yeast 22:11551169

Wang Y, Wang H, Feng X, Wang X, Huang J (2010) Biohydrogen production from cornstalk wastes by anaerobic fermentation with activated sludge. Int J Hydrogen Energy 35:3092-3099
Werner JJ, Knights D, Garcia ML, Scalfone NB, Smith S, Yarasheski K, Cummings TA, Beers AR, Knight R, Angenent LT (2011) Bacterial community structures are unique and resilient in full-scale bioenergy systems. Proc Natl Acad Sci 108:4158-4163

Winder CL, Dunn WB, Schuler S, Broadhurst D, Jarvis R, Stephens GM, Goodacre R (2008) Global metabolic profiling of Escherichia coli cultures: an evaluation of methods for quenching and extraction of intracellular metabolites. Anal Chem 80:2939-2948

Xia J, Mandal R, Sinelnikov IV, Broadhurst D, Wishart DS (2012) MetaboAnalyst 2.0-a comprehensive server for metabolomic data analysis. Nucleic Acids Res 40:W127W133 